\title{
Cathodoluminescence Profiling of InGaN-Based Quantum Well Structures and Laser Diodes - In-Plane Instabilities of Light Emission
}

\author{
M. Godlewski ${ }^{a, b}$, V.Yu. Ivanov ${ }^{a}$, E.M. Goldys ${ }^{c}$, \\ M. Phillips ${ }^{d}$, T. Böttcher ${ }^{e}$, S. FigGE $^{e}$, D. Hommé ${ }^{e}$, \\ R. Czernecki ${ }^{f}$, P. Prystawko ${ }^{f}$, M. Leszczynski ${ }^{f}$, \\ P. Perlin ${ }^{f}$, I. Grzegory ${ }^{f}$ And S. Porowski ${ }^{f}$ \\ ${ }^{a}$ Institute of Physics, Polish Academy of Sciences \\ al. Lotników 32/46, 02-668 Warsaw, Poland \\ ${ }^{b}$ College of Science, Cardinal S. Wyszyński University, Warsaw, Poland \\ ${ }^{c}$ Division of Information and Communication Sciences \\ Macquarie University, Sydney, Australia \\ ${ }^{d}$ Microstructural Analysis Unit, UTS, Sydney, Australia \\ ${ }^{e}$ Institute of Solid State Physics, University of Bremen, 28334 Bremen, Germany \\ ${ }^{f}$ High Pressure Res. Center (Unipress), Polish Academy of Sciences \\ Warsaw, Poland

\begin{abstract}
Instabilities of light emission and also of stimulated emission in series of GaN epilayers and InGaN quantum well structures, including laser diode structures, are studied. A stimulated emission is observed under electron beam pumping. This enabled us to study light emission properties from laser structures and their relation to microstructure details. We demonstrate large in-plane fluctuations of light emission and that these fluctuations are also present for excitation densities larger than the threshold densities for the stimulated emission.
\end{abstract}

PACS numbers: 61.72.Ff, 61.72.Mm, 68.37.Hk, 78.60.Hk 


\section{Introduction}

Basov et al. first demonstrated (for liquid xenon) the possibility of generating laser radiation by excitation with an electron beam [1]. Several groups reported then electron beam pumped laser action in gas and liquid media. The concept became practical with an introduction of low voltage field emission microtips and by using semiconductor-based heterostructures. For example, blue laser light emission from $\mathrm{ZnCdSe/ZnSe} \mathrm{heterostructures} \mathrm{was} \mathrm{demonstrated} \mathrm{[2].} \mathrm{It} \mathrm{was} \mathrm{demonstrated}$ that microgun pumped lasers avoid limitations of $p$-type doping of ZnSe [2]. Doping of the active layer of laser reduces the threshold pump current only in the case of short nonradiative lifetimes in the device [3]. Otherwise, we can use undoped structures.

Another advantageous property is that the laser action can often be achieved at record low threshold currents [4]. Threshold currents as low as $5 \mathrm{~A} / \mathrm{cm}^{2}$ (for $\mathrm{ZnSe}$ ), $12 \mathrm{~A} / \mathrm{cm}^{2}$ (for $\mathrm{ZnSe} / \mathrm{ZnSSe}$ superlattice) [5] and $3 \mathrm{~A} / \mathrm{cm}^{2}$ [6] were reported. Lowering of a threshold current helps to eliminate some of degradation processes accounted in semiconductor-based laser devices [7].

Laser action under electron beam pumping was achieved in very different media and structures: for example for advanced laser structures, such as VCSEL structures [8], but for single quantum well (QW) and double QW GRINSCH structures with $0.5-1 \mathrm{~mm}$ long microcavities [2], as well. Importantly, laser action was also reported in very simple structures, such as in samples covered with metal mirrors [9] or even from large area single crystals [10]. Laser modes were reported only in the former case [2]. In all cases the method turned out to be very useful also in studying degradation mechanisms in semiconductor based structures [11, 12].

In this paper we report observation of a stimulated emission in QW structures of $\mathrm{GaN} / \mathrm{InGaN}$. This observation allows us to evaluate in-plane instabilities of stimulated emission and to relate them to microstructure details of the samples studied.

\section{Samples and experimental techniques}

The samples have been grown by metalorganic vapor phase epitaxy (MOVPE) on sapphire (heterostructures) or bulk GaN substrate (homoepitaxial samples). Two series (each of four samples) of heteroepitaxial GaN epilayers were studied. These samples were grown on sapphire/GaN templates with dislocation density of $1 \times 10^{10} \mathrm{~cm}^{-2}$ (g411 series) or $2 \times 10^{9} \mathrm{~cm}^{-2}$ (g457 series). Each series consisted of template and of three epilayers with $1500 \mathrm{~nm}$ thickness. One of them was undoped, two other were $n$-type doped with Si to the level $10^{18} \mathrm{~cm}^{-3}$ and $10^{19} \mathrm{~cm}^{-3}$.

Homoepitaxial samples have the highest structural quality achievable at present, with densities of dislocations typically below $10^{4} \mathrm{~cm}^{-2}$. Two series $(511$ and 534) of homoepitaxial QW structures were studied. Each of them contained 
an active region consisting of $20 \mathrm{InGaN}$ QWs with GaN barriers. Indium fraction was about $2 \%$ in 511 series and $2.8-3 \%$ in series 534 . The structures had a different width of QW and barrier regions - $2.8 \mathrm{~nm}(\mathrm{QW}) / 5.3 \mathrm{~nm}$ (barrier) for 511 series and $4 \mathrm{~nm}(\mathrm{QW}) / 8 \mathrm{~nm}$ (barrier) in series 534. Growth temperature was $790^{\circ} \mathrm{C}$ for the 511 series and $785^{\circ} \mathrm{C}$ for the 534 series. Structures were grown on GaN bulk substrate covered with GaN buffer layer ( $1 \mu \mathrm{m}$ thick in 534 series, $0.55 \mu \mathrm{m}$ thick in 511 series). Samples varying in structural quality were selected to get better insight into origin of the observed emission instabilities.

Scanning electron microscopy (SEM) and cathodoluminescence (CL) measurements were performed at room temperature using a JEOL JSM-6300F scanning microscope with a field emission gun (for SEM) and JEOL 35C electron microscope, equipped with MonoCL2 setup of Oxford Instruments for CL studies.

\section{Results and discussion}

It was reported that photoluminescence of $\mathrm{GaN}$ and InGaN epilayers and QW structures is significantly enhanced upon $n$-type doping [13-15]. The effect was tentatively explained by screening of piezoelectric fields, improvement of morphology (smoother interfaces) or by saturation of deep dislocation-related centers, expected in heavily doped samples. The latter limits role of dislocations as centers of nonradiative recombination. To verify such possibility we performed CL studies of a series of GaN epilayers of a different dislocation density and doping level. CL intensity upon varying excitation density was measured. The results are shown in Fig. 1. To the relatively large current (10 nA) CL intensity increases nonlinearly, but relatively slowly. Such observation was in line with previous data and could suggest that in fact we compensate/saturate centers of nonradiative recombination.

A rapid jump in the CL intensity was observed for current above $9-10 \mathrm{nA}$ (Fig. 1). This suggested that we reached threshold current to excite a stimulated emission, but the explanation was only tentative, since we observed the effect only

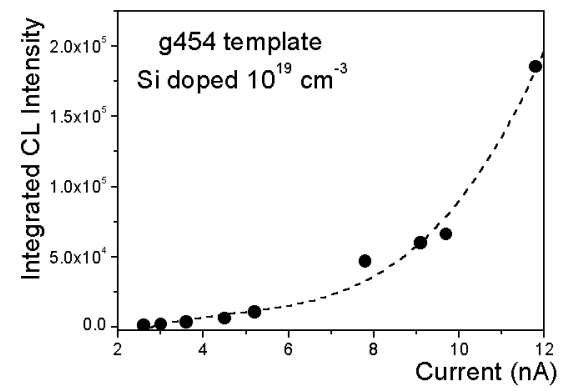

Fig. 1. Dependence of the CL intensity on excitation current in CL study of Si doped GaN epilayer grown by MOVPE on GaN/sapphire template with $2 \times 10^{9} \mathrm{~cm}^{-2}$ dislocations. 
for heavily $n$-type doped epilayer grown on template with a lower (but still relatively high) dislocation density. To verify such possibility, and also to use it for studying CL instabilities, we employed CL for a series of homoepitaxial samples with a high structural quality. We used similar structures to the one for which we reported record low threshold power for optical pumping of a stimulated emission $[16]$.

The results of CL studies of homoepitaxial samples are shown in Fig. 2. Three of five samples studied showed a threshold-like dependence on beam current density, which we explain by a stimulated emission from the films. The most intensive (with the smallest threshold) CL emission was observed for the sample $534 \mathrm{~A}$. For this sample we also observed a large homogeneity of CL emission, i.e., electron beam density dependence is similar, when measured at different areas of the film.

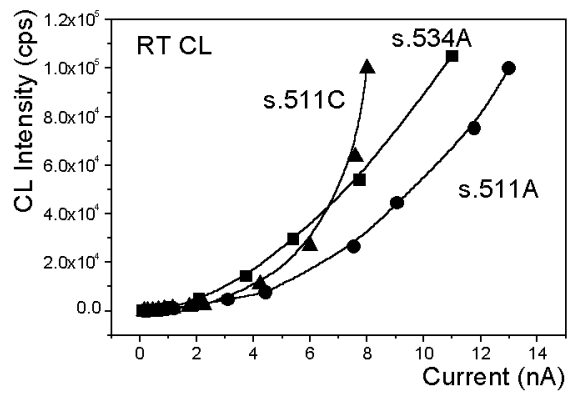

Fig. 2. Threshold density dependences measured for three InGaN/GaN homoepitaxial structures at 2000 magnification, $10 \mathrm{kV}$ accelerating voltage and at room temperature.

As mentioned in the introduction, structures pumped with electron beam often show record low threshold densities. We can evaluate the threshold density in our system by taking as an excitation radius, radius of a cloud of primary and secondary electrons. This radius is fairly small, of about $50 \mathrm{~nm}$, since diffusion of carriers and excitons is limited by strong localization effects. The estimated threshold density is then in the range of $100 \mathrm{~A} / \mathrm{cm}^{2}$, i.e., is fairly low. Problems with $p$-type doping and with contacts technology lead to much larger threshold densities in laser structures operating under current injection conditions.

After selecting structures showing a stimulated emission we studied their morphology and relation between morphology and in-plane fluctuations of the QW emission. The relevant results are shown in Figs. 3 and 4 . We found a clear correlation between CL properties and structural quality of the structures. The samples showing the lowest threshold densities show the best structural quality. Density of pinholes and dislocations is low. Atomic size growth steps are observed.

Figure 4 shows in-plane fluctuations of the QW stimulated emission on the example of the $511 \mathrm{C}$ structure. The observed CL fluctuations directly reflect 

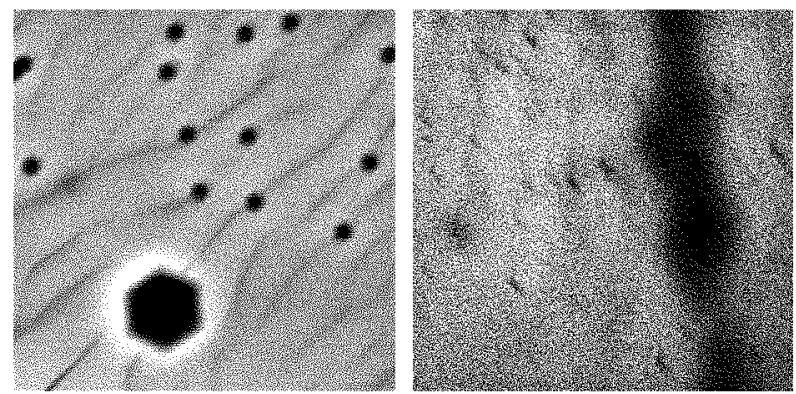

Fig. 3. SEM images taken at $10 \mathrm{kV}$ accelerating voltage and at 20000 magnification (from $5 \times 5 \mu \mathrm{m}$ region) for $534 \mathrm{~A}$ (left) and $511 \mathrm{C}$ (right) $\mathrm{GaN} / \mathrm{InGaN}$ homoepitaxial QW structures.

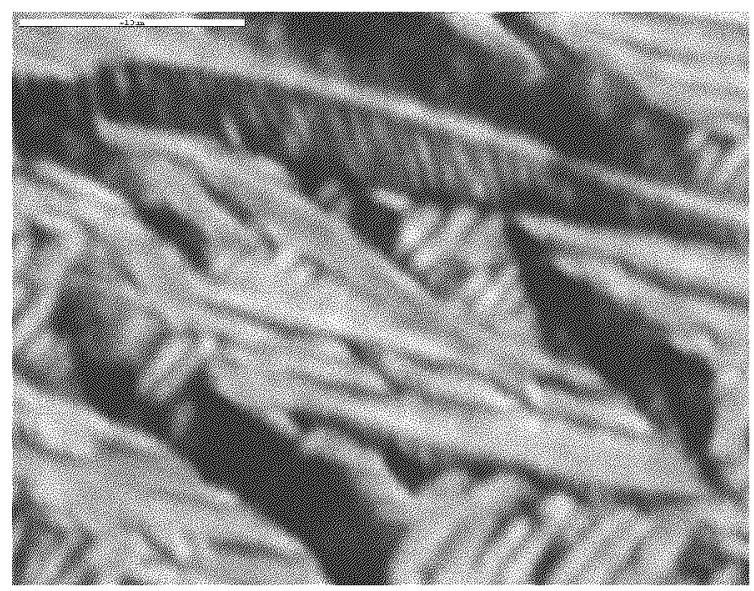

Fig. 4. Scanning CL images taken with detection set at the edge CL, at $10 \mathrm{kV}$ accelerating voltage and at 6000 magnification, using $1.8 \mathrm{nA}$ excitation current (threshold density).

growth details. Atomic size growth steps, as observed in the SEM, are even more pronounced in the scanning CL study. We can thus claim that there is a direct correlation between structural quality of the films and light emission properties. CL fluctuates in the intensity for excitation below and above threshold density. The observed fluctuations reflect microstructure of the sample and not e.g. indium fraction fluctuations, which typically are of a much smaller scale.

\section{Conclusions}

We report observation of a stimulated emission from homoepitaxial GaN/InGaN QW structures. Stimulated emission is observed only for the structures of a good structural quality and shows in-plane fluctuations of intensity, which directly correlate to microstructure of the structures. 


\section{Acknowledgments}

This work was partly supported by the grant number 5 P03B 00720 of the State Committee for Scientific Research (Poland) for the years 2001-2003 and by the DENIS program of European Union (G5RD-CT-2001-00566).

\section{References}

[1] N.G. Basov, V.A. Danilychev, Yu. M. Popov, Kvantovaya Elektronika 1, 29 (1971).

[2] D. Herve, R. Accomo, E. Molva, L. Vanzetti, J.J. Paggel, L. Sorba, A. Franciosi, Appl. Phys. Lett. 67, 2144 (1995).

[3] B.M. Lavrushin, R.F. Nabiev, Yu.M. Popov, Kvantovaya Elektronika 15, 78 (1988).

[4] B. Fitzpatric, J. Khurgin, P. Harnack, D. de Leeuw, in: Int. Electron Devices Meeting, 1986, Technical Digest (Cat. No. 86CH2381-2, New York) IEEE 1986, p. 630.

[5] D.A. Cammack, R.J. Dalby, H.J. Cornelissen, J. Khurgin, J. Appl. Phys. 62, 3071 (1987).

[6] B.J. Fitzpatrick, P.M. Harnack, S. Cherin, Philips J. Res. 41, 452 (1986).

[7] V.N. Katsap, V.I. Kozlovskii, V.Yu. Kruchnov, A.V. Namm, A.S. Nasibov, V.B. Nosikov, P.V. Reznikov, V.N. Ulasyuk, Kvantovaya Elektronika 14, 1994 (1987).

[8] A. Onischenko, J. Surma, SPIE-Int. Soc. Opt. Eng. Proc. SPIE 3211, 126 (1999).

[9] V.P. Gribkovskii, A.L. Gurskii, S.V. Davydov, E.V. Lutsenko, I.I. Kulak, A.I. Mitkovets, G.P. Yablonskii, V.F. Gremerik, Jpn. J. Appl. Phys. 32, Suppl. 3, 521 (1993).

[10] B.J. Fitzpatrick, T.F. McGee III, P.M. Harnack, J. Cryst. Growth 78, 242 (1986).

[11] J.-M. Bonard, J.-D. Ganiere, La Vanzetti, J.J. Paggel, L. Sorba, A. Franciosi, D. Herve, E. Molva, J. Appl. Phys. 84, 1263 (1998).

[12] L.V. Borkovskaya, B.R. Dzhumaev, N.E. Korsunskaya, V.P. Papusha, G.S. Pekar, A.F. Singaevsky, SPIE-Int. Soc. Opt. Eng. Proc. SPIE 3724, 244 (1999).

[13] S. Nakamura, G. Fasol, The Blue Laser Diode, Springer Verlag, Berlin 1997.

[14] S. Nakamura, T. Mukai, M. Senoh, Jpn. J. Appl. Phys. (Part 2) 32, 16 (1993).

[15] S. Nakamura, M. Senoh, S. Nagahama, N. Iwasa, T. Yamada, T. Matsushita, Y. Sugimito, H. Kiyoku, Appl. Phys. Lett. 70, 1417 (1997).

[16] V.Yu. Ivanov, M. Godlewski, H. Teisseyre, P. Perlin, R. Czernecki, P. Prystawko, M. Leszczynski, I. Grzegory, T. Suski, S. Porowski, Appl. Phys. Lett. 81, 3735 (2002). 